\title{
Resonant processes as a tool for revealing universe's hidden dimensions
}

\author{
Alexander A. Antonov ${ }^{1 *}$ \\ ${ }^{1}$ Research Center of Information Technologies "Telannet Electronics" \\ *Correspondence author: tel/fax (380) 444243587; e-mail: telan@bk.ru
}

\begin{abstract}
It has been theoretically and experimentally proven that complex frequencies, immitance functions of complex frequencies, as well as resonance at complex frequencies are physically real. It follows from the above that the physical macrocosm has extra hidden dimensions. The information expands the fundamental knowledge on the structure of the Universe and may lead to reconsideration of certain basic concepts in mathematical, physical and engineering sciences.
\end{abstract}

Key words: resonance, real frequencies, complex frequencies, extra dimensions, hidden dimensions, Universe

\section{INTRODUCTION}

Resonance as a physical phenomenon has been long known in science and is widely used in engineering. It is especially convenient to study it in radio engineering and electrical engineering where any situation can be easily implemented. There are numerous publications on the theory of electric circuits (Bode 1945; Steinmetz 2006; Bell 2009), which offer analysis of the processes in these circuits using both real and complex frequencies. However, no account has been given of the physical meaning of complex frequencies, as well as of other concrete complex numbers (resistance, voltage drop, power, energy, etc.) depending on complex frequency. There is also no explanation of whether resonance can exist at complex frequencies, despite the huge physical importance of the issue.

Resonance at real frequencies : As is well known, in linear electric circuits resonance is understood as a phenomenon, which, as the real frequency of the external impact approaches the real resonant frequency, results in the following:

- a phase shift between the external impact and the forced component of the response comes down to zero;

- the amplitude of the forced component of the response takes an extreme value;

- the frequency of free oscillations becomes equal to the resonant frequency.

However, it turns out that the resonance at real frequencies having all the above characteristics can take place only in electric LC-circuits, which are of limited practical interest. In real electric LCR-circuits this is achieved only as the result of the simplified analysis of resonance, which is exactly what is performed everywhere. If precise mathematical analysis of resonance in LCR-circuits is performed, it turns out that the frequencies corresponding to the above characteristics of resonance are different, although only marginally (however, in physics it is not acceptable to ignore a new phenomenon for such a reason). Moreover, the above manifestations of resonance take place not even at one, but at several different real resonance frequencies. In order to make sure this is true, let us consider the simplest example.

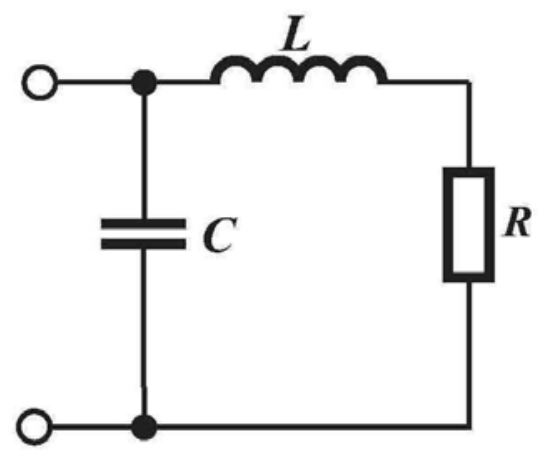

Fig. 1 The electric LCR-circuit under consideration.

The conductivity of the electric LCR-circuit given in Fig. 1, is

$$
Y(j \omega)=C \frac{\left(\frac{1}{L C}-\omega^{2}\right)+j \omega \frac{R}{L}}{\frac{R}{L}+j \omega}=L \frac{\left(\omega_{0}^{2}-\omega^{2}\right)+j \omega 2 \sigma_{0}}{2 \sigma_{0}+j \omega}
$$

where $2 \sigma_{0}=R / L ; \omega_{0}=1 / \sqrt{L C}$. 
The reactive component of this complex conductivity (see the solid line in Fig. 2) is

$$
\operatorname{Im} Y(j \omega)=C \frac{\omega\left(\omega^{2}-\omega_{0}^{2}+4 \sigma_{0}^{2}\right)}{\omega^{2}+4 \sigma_{0}^{2}}
$$

Setting it to zero, we get the equation, the solution of which yields the combination of two (not one!) resonance frequencies corresponding to the first attribute of resonance discovered during the analysis of LC-circuits

$$
\left[\begin{array}{l}
\omega_{r e s 1}^{\prime}=0 \\
\omega_{r e s 1}^{\prime \prime}=\sqrt{\omega_{0}^{2}-4{\sigma_{0}^{2}}^{2}}=\omega_{0} \frac{\sqrt{Q^{2}-1}}{Q}
\end{array}\right.
$$

where $Q=\frac{\omega_{0}}{2 \sigma_{0}}=\frac{1}{R} \sqrt{\frac{L}{C}}$ is the $Q$ factor.

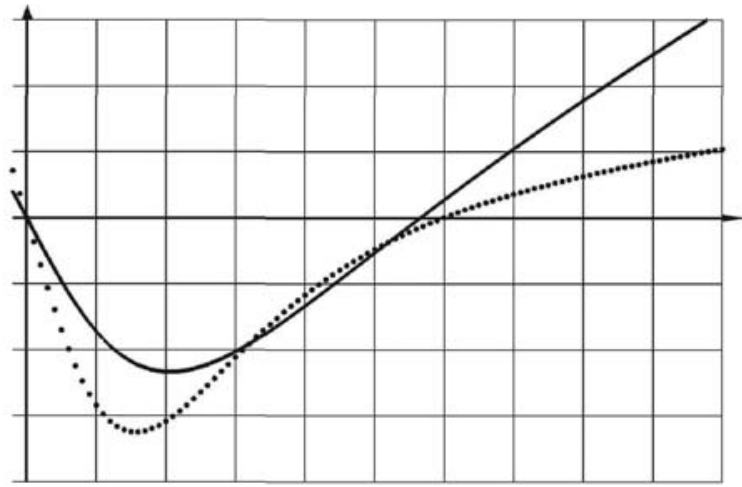

$\omega$

Fig. 2 Graphs of the dependencies for determining resonance frequencies.

The absolute value of complex conductivity of this oscillating LCR-circuit is

$$
|Y(j \omega)|=\tilde{N} \sqrt{\frac{\left(\omega_{0}^{2}+\omega^{2}\right)^{2}+4 \sigma_{0}^{2} \omega^{2}}{\omega^{2}+4 \sigma_{0}^{2}}}
$$

As a result of extremum analysis of the radicand, i.e., the solution of the equation (see the dotted line on Fig. 2)

$$
\begin{aligned}
& \frac{d}{d \omega}\left[\frac{\left(\omega_{0}^{2}-\omega^{2}\right)^{2}+4 \sigma_{0}^{2} \omega^{2}}{\omega^{2}+4 \sigma_{0}^{2}}\right]= \\
& =\frac{2 \omega\left[\omega^{4}+8 \sigma_{0}^{2} \omega^{2}-\left(\omega_{0}^{4}+8 \sigma_{0}^{2} \omega_{0}^{2}-16 \sigma_{0}^{4}\right)\right]}{\left(\omega^{2}+4 \sigma_{0}^{2}\right)^{2}}=0
\end{aligned}
$$

we get another combination of two (once again!) solutions corresponding to the second attribute of resonance discovered during the analysis of LC-circuits

$$
\left[\begin{array}{l}
\omega_{\text {res } 2}^{\prime}=0 \\
\omega_{\text {res } 2}^{\prime \prime}=\sqrt{\omega_{0} \sqrt{\omega_{0}^{2}+8 \sigma_{0}^{2}}-4 \sigma_{0}^{2}}= \\
=\omega_{0} \frac{\sqrt{Q \sqrt{Q^{2}+2}-1}}{Q}
\end{array}\right.
$$

As can be seen (see Fig. 2), for the given LCRcircuit we eventually obtained three different

\begin{tabular}{|c|c|c|c|}
\hline $\lg \mathrm{Q}$ & $Q$ & $\omega_{r e s 1}^{\prime \prime} / \omega_{0}$ & $\omega_{\text {res } 2}^{\prime \prime} / \omega_{0}$ \\
\hline 0 & 1,000000000 & 0,000000000 & 0,855599677 \\
\hline 0,1 & 1,258925412 & 0,607488811 & 0,934349493 \\
\hline 0,2 & 1,584893192 & 0,775817523 & 0,970629714 \\
\hline 0,3 & 1,995262315 & 0,865338868 & 0,987181020 \\
\hline 0,4 & 2,511886432 & 0,917338913 & 0,994538877 \\
\hline 0,5 & 3,162277660 & 0,948683298 & 0,997719958 \\
\hline 0,6 & 3,981071706 & 0,967938151 & 0,999062537 \\
\hline 0,7 & 5,011872336 & 0,979892485 & 0,999618734 \\
\hline 0,8 & 6,309573445 & 0,987360691 & 0,999846091 \\
\hline 0,9 & 7,943282347 & 0,992043884 & 0,999938177 \\
\hline 1,0 & 10,000000000 & 0,994987437 & 0,999975247 \\
\hline 1,1 & 12,589254118 & 0,996840221 & 0,999990110 \\
\hline 1,2 & 15,848931925 & 0,998007479 & 0,999996053 \\
\hline 1,3 & 19,952623150 & 0,998743267 & 0,999998427 \\
\hline 1,4 & 25,118864315 & 0,999207239 & 0,999999373 \\
\hline 1,5 & 31,622776601 & 0,999499875 & 0,999999750 \\
\hline 1,6 & 39,810717055 & 0,999684472 & 0,999999801 \\
\hline 1,7 & 50,118723363 & 0,999800927 & 0,999999957 \\
\hline 1,8 & 63,095734480 & 0,999874398 & 0,999999984 \\
\hline 1,9 & 79,432823472 & 0,999920752 & 0,999999994 \\
\hline 2,0 & 100,000000000 & 0,999949999 & 0,999999998 \\
\hline
\end{tabular}
resonance frequencies, as $\omega_{\text {res } 1}^{\prime}=\omega_{\text {res } 2}^{\prime}=0$, and $\omega_{\text {res } 1}^{\prime \prime} \neq \omega_{\text {res } 2}^{\prime \prime} \neq 0$.

Table contains the results of calculations by means formulae (1) and (2).

Table Sizes of resonant frequencies

It is undifficult to notice (see the table) that the aforesaid differences between resonant frequencies and $\omega_{0}$ value are rather small and usually do not exceed the experiment error. That's why many people even do not suspect of such differences. 
Similar results are obtained after analyzing other LCR-circuits, as well, both the two-terminal and the two-terminal-pair networks, both of the second degree and of a higher degree.

Moreover, the frequency of free oscillations $\omega_{\text {free }}=\sqrt{\omega_{0}^{2}-\sigma_{0}^{2}}$ in the electric LCR-circuits, contrary to the electric LC-circuits, always turns out to equal none of the resonance frequencies. Mandelstam (1955) pointed to the inexplicability of this circumstance back.

Thus, taking into account the above inconsistencies of resonance explanation at real frequencies, it has to be recognized as imperfect.

Resonance at complex frequencies: In the theory of analysis and synthesis of electric LCR-circuits, apart from the concept of real frequency, the concept of complex frequency is also widely used and explained by various authors in a different way. However, these differences in explaining complex frequency are not relevant right now. We can use any.

Another fact is important. The immitance functions of complex frequency obtained for a particular LCRcircuit (which are understood as resistances, or conductivity, or transfer functions) take zero or infinitely large values of magnitude at certain values of the complex frequency. This occurs because both the numerator and the denominator of the fractional rational function are certain polynomials of the complex frequency. Thus, setting to zero the polynomial in the numerator and solving the corresponding algebraic equation, we can find the values of the so-called 'zeros'. Setting to zero the polynomial in the denominator and solving the corresponding algebraic equation, we can find the values of the so-called 'poles'.

For the simplest case, when the external impact applied to the electric circuit is made by continuous sinusoidal oscillations, the term 'resonant frequency' is used instead of the above 'zeros' and 'poles'. Respectively, the processes taking place at these oscillations are referred to as resonant processes.

Thus, complex 'zeros' and 'poles' would seemingly have to be called resonant frequencies, as well. Sometimes they are indeed referred to like that (Dolginov 1957; Antonov 1987). However, most experts in the theory of electric circuits do not use the term 'resonant frequency' with reference to 'zeros' and 'poles', because 'zeros' and 'poles' are complex numbers. Thus, if we admit the term 'complex resonant frequency', we will have to admit the existence of resonance at complex frequencies and the physical reality of complex frequencies themselves. After all, we will have to study this resonance at complex frequencies and prove its existence.

And it does exist, because, as will be shown below, the interpretation of resonance at complex frequencies:

- is theoretically self-consistent and fully corresponds to all attributes of resonance;

- allows explaining the inconsistencies of explaining resonance at real frequencies;

- is proven experimentally.

It is easy to prove this theoretically, because it is the above 'zeros' and 'poles' that are understood as resonant complex frequencies, with all the consequences that come with it. The same 'zeros' and 'poles' also correspond to the complex-conjugate frequencies of free oscillations.

It is also easy to make sure that the above selfconsistent resonance in electric LC-circuits at real frequencies is, in fact, just a particular case of resonance at complex frequencies.

The use of vector diagrams at complex frequencies (Antonov 2009) allows giving an exhaustive explanation of why several real resonant frequencies appear during the investigation of resonance at real frequencies.

Thus, the interpretation of resonance at complex frequencies is convincing and self-consistent, contrary to the interpretation of resonance at real frequencies.

It is somewhat more difficult to offer experimental evidence of resonance at complex frequencies (Antonov 2008, 2009, 2010a,b), because in order to do this we will have to minimize the influence of the free component of the response, which interferes into the observations. 

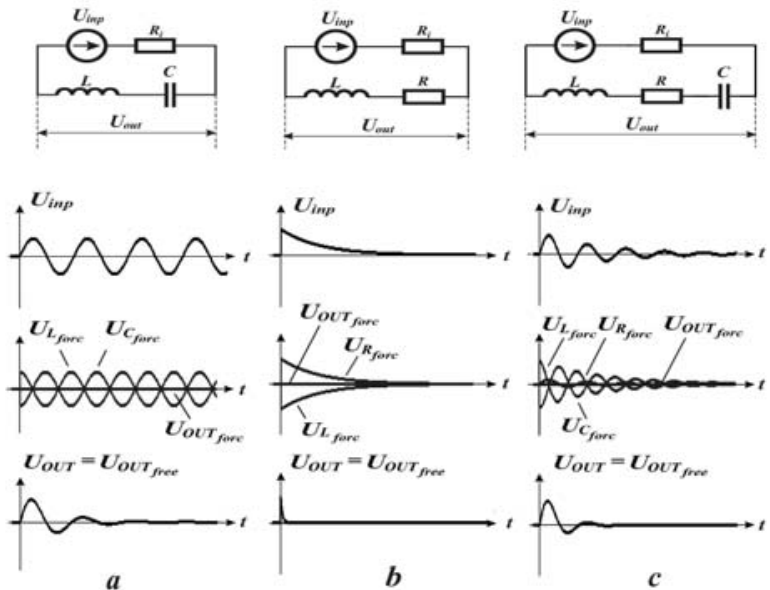

Fig. 3 Resonance in LC- , LR- and LCR-two-terminals.

This circumstance deserves special explanation. Let us recollect with this respect the well-known but sometimes omitted fact that, for resonance at real frequencies, the forced and free components of the response are separated on their own accord in the course of time. Indeed, the transitional process in stable electric LCR-circuits always fades out in the course of time. The forced component of the response to the incoming undamped oscillations constitutes undamped oscillations. Consequently, after a certain period corresponding to the duration of the transitional process the observer always has the possibility to register only forced oscillations. This is the mode usually used to study LCR-circuits.

During the investigation of resonance at complex frequencies, the impact on the electric circuit under investigation is made by exponential radio pulses (if $p=-\sigma \pm j \omega$ ) or video pulses (if $p=-\sigma$ ), the duration of which is time-constrained. Therefore, their duration may turn out to be comparable with that of the transitional processes. Thus, to make the observation more convenient, these impacts should be repeated. However, this alone is not enough. It is also necessary somehow to reduce the free component of the response, which interferes into the observation of the forced component. This can be achieved, for instance, by significantly reducing the magnitude of the free component of the response by modifying the initial conditions (Antonov 1970). It is also possible to calculate the parameters of the circuit under investigation in such a way that the forced and the free components of the response would be significantly (e.g., by an order of magnitude) different in duration.

The latter technique is used in the experiment the results of which are presented in Fig. 3. As can be seen, when an LCR-two-terminal is impacted by exponential radio pulses (see Fig. 3c), the forced component of the response $U_{O U T_{\text {for }}}$ at the complex resonant frequency $p_{\text {res }}=-\sigma_{0} \pm j \sqrt{\omega_{0}^{2}-\sigma_{0}^{2}}$ takes zero value, and only the free component of the response $U_{\text {OUT free }_{\text {fre }}}$ can be observed at the output. That is, we have the result fully corresponding to all attributes of resonance.

In this respect, it is interesting to note that if the same LCR-two-terminal is impacted by the undamped oscillations, it is impossible to get zero value of the forced component of the response $U_{O U T_{\text {forc }}}$ at any frequency of the impact $p= \pm j \omega$. Therefore, in this case there is no resonance, and the process taking place in the LCR-circuit is simply a near-resonant (or quasi-resonant) one.

Similarly, if the LCR-two-terminal is impacted by exponential video pulses (see Fig. 3b), the forced component of the response $U_{O U T_{\text {forc }}}$ at complex resonant frequency $p_{\text {res }}=-\sigma_{0}$ also takes zero value, and at the output only the free component of the response $U_{O U T_{\text {free }}}$ is observed. That is, resonance takes place in this case, as well.

Finally, at complex resonant frequency $p_{\text {res }}= \pm j \omega_{0}$, i.e., if the LC-two-terminal is impacted by undamped sinusoidal oscillations (see Fig. 3a), the forced component of the response $U_{\text {OUT }_{\text {orr }}}$ takes zero value, and the voltage drop at it contains only the free component $U_{O U T_{\text {free }}}$. This is why the well-known resonance in the LC-circuit is observed.

Thus, using the concept of complex frequency, all of the above electric circuits turned out to be resonant ones, however, for different corresponding impacts and at different complex resonant frequencies.

The experiments above are simple and available to any engineer. The usual equipment in any laboratory allows verifying them, as well as performing other similar experiments.

There is other evidence of physical reality of complex numbers, as well (Antonov 2010c).

Thus, the above investigations allow stating that the existence of resonance at complex frequencies has 
been implicitly proven both theoretically and experimentally.

Revealing the Universe's extra dimensions: It follows from physical reality of resonance at complex frequencies that the complex frequencies themselves are physically real. Therefore, not only real components of them are physically real, but their imaginary components, too, especially since both of these components have a similar influence on the process of resonance (see Fig. 3).

Yet, after all the explanations and evidence given above, there is still the desire to get the answer to the following question - how is it possible to see the real and the imaginary components of the complex frequency? Seeing is particularly important, as people think with visual images.

However, it is impossible to see the frequency itself, not only the complex one, but the real one, either, just as we cannot see, for instance, the time. Nevertheless, it is possible to see the influence of frequency on the oscillation process. To this end, it is necessary to project the damped sinusoidal oscillations on an oscillograph screen. According to the Euler formula, one of the parameters of the complex frequency of these oscillations determines the damping rate, whereas the other defines the frequency of crossing the time axis.

However, it follows from physical reality of concrete complex numbers that many other physical quantities are also physically real, such as complex resistance, complex voltage, and other quantities, which are a function of complex frequency. Moreover, since damped oscillations can be not only electromagnetic, but also mechanic, acoustic, hydraulic, etc., the corresponding complex parameters of these oscillations are also physically real.

Is it possible to see them? No. For some of them, such as voltage, resistance, power, and others, it is impossible to see either the imaginary or the real components. For others, such as distance in case of observing the mechanic oscillations, it is possible to see the real component, but not the imaginary one.

In this respect, it would be good to recollect that many other physically real natural phenomena, such as the magnetic field, the X-rays, the elementary particles, the black holes, and many others, are invisible for us, as well. Nevertheless, we admit that they are physically real, based on the theoretical and experimental evidence presented by scientists.
On the same basis, due to the irrefutable evidence given above, we have to admit the physical reality of concrete complex numbers, although we will never be able to see their imaginary component.

One more question may arise. If the concrete complex numbers are physically real, do they actually measure anything? And on this basis, can the imaginary components of concrete complex numbers be considered as some extra dimensions in addition to the dimensions measured by real numbers? Certainly yes, because at least certain imaginary components of concrete complex numbers (e.g. those of voltage, power, etc.) can already be measured with the equipment available to scientists. However, since we are not able to perceive any imaginary components of concrete complex numbers with our senses, they can be referred to as hidden dimensions, by analogy with the hidden dimensions described in (Randall 2005) and which are expected to be discovered during the experiments at the Large Hadron Collider.

However, the hidden dimensions revealed and described in the paper are different, as the Large Hadron Collider studies the microcosm, whereas the paper deals with the processes in the macrocosm. Certainly, we do not rule out that realizing the physical reality of concrete complex numbers can turn out to be useful for the research of the microcosm, as well.

\section{CONCLUSIONS}

Based on the results of investigating the resonant processes, the Universe's hidden dimensions have been revealed. Although resonant processes of any physical nature can be used as the object of research, we describe resonant processes in the electric LCR-circuits, since they are most convenient for observation.

The research has shown that resonant processes in the electric LCR-circuits take into account the magnitude of complex (but not real) frequencies of the input action and the free component of the response. This proves the physical reality of complex frequencies, the parameters of electric circuits at complex frequencies, and resonance at complex frequencies. The results are also true for oscillation processes of any other physical nature.

However, the physically real quantities mentioned above are complex only during the time of damped oscillations, because it is only during this time that they acquire the additional components represented 
by imaginary numbers. And it is only during this time that the corresponding extra dimensions open up in our physical world. These extra dimensions, since people do not even suspect their existence, can be referred to as hidden dimensions, similar to the extra hidden dimensions which are expected to be discovered at the Large Hadron Collider.

Finally, we do not rule out that, apart from the extra dimensions discussed in (Randall 2005) and in this paper, there can be other extra dimensions, for instance, those corresponding to the physically real hypercomplex numbers (Kantor \& Solodovnikov 1989 ), of course, if their physical reality is proven.

\section{REFERENCES}

Antonov, A.A. \& Bazhev, V.M. 1970 Patent of the USSR № 433650.

Antonov, A.A. 1987 Investigation of Resonance. Preprint № 67 of the Institute for Modelling in Energy Engineering of AS of the UkrSSR (Kiev).

Antonov, A.A. 2009 Resonance on Real and Complex Frequencies. Eur. J. Sci. Res. 28.2, 193-204.

Antonov, A.A. 2008 Physical Reality of Resonance on Complex Frequencies. Eur. J. Sci. Res. 21.4, 627-641.
Antonov, A.A. 2010a New Interpretation of Resonance. Int. J. Pure Appl. Sci. Technol. 1.2 1-12.

Antonov, A.A. 2010b Oscillation Processes as a Tool of Physics Cognition. Amer. J. Sci. Ind. Res. 1.2, $342-$ 349. (doi:10.5251/ajsir.2010.1.2.342.349)

Antonov, A.A. 2010c Solution of Algebraic Quadratic Equations Taking into Account Transitional Processes in Oscillation Systems. Gen. Math. Notes. 1.2, 11-16.

Bell, D.A. 2009 Fundamentals of Electric Circuits (Oxford University Press, Oxford, UK).

Bode, H.W. 1945 Network Analysis and Feedback Amplifier Design (Van Nostrand, Princeton, UK).

Dolginov, A.I. 1957 Resonance in Electric Circuits and Systems (Gosenergoizdat, Moscow).

Kantor, I.L. \& Solodovnikov, A.S. 1989 Hypercomplex Numbers (Springer-Verlag, Berlin).

Mandelshtam, L.I. 1955 Lectures on Theory of Oscillations, Vol.4. (Publishing House of AS of the USSR, Moscow).

Randall, L. 2005 Warped Passages: Unraveling The Mysteries Of The Universe's Hidden Dimensions (Ecco, NY).

Steinmetz, C.P. 2006 Theory and Calculation of Electric Circuits (Wexford College Press). 